\title{
Creating and probing of a perfect vortex in situ with an optically trapped particle
}

\author{
Mingzhou Chen, ${ }^{1 *}$ Michael Mazilu, ${ }^{1}$ Yoshihiko Arita, ${ }^{1}$ Ewan M. Wright ${ }^{1,2}$ and Kishan \\ Dholakia $^{1,2}$
}

${ }^{1}$ SUPA, School of Physics \& Astronomy, University of St Andrews, North Haugh, St Andrews, KY16 9SS, United Kingdom

${ }^{2}$ College of Optical Sciences, The University of Arizona, 1630 East University Boulevard, Tuscon, Arizona 85721-0094, USA.

We experimentally create a 'perfect' vortex beam, which has a uniform ring profile and fixed radius. In contrast with other vortex fields, the beam profile is independent of its topological charge. We then correct this field in situ using a single trapped particle as a probe. This results in a constant angular velocity for the particle regardless of its position on the beam circumference.

Keywords: Optical vortices, Optical manipulation, Optical tweezers

\section{Introduction}

Light fields that have a singular point - a vortex have created widespread interest across many fields. Intriguingly, an optical vortex beam may possess orbital angular momentum $(\mathrm{OAM})^{1)}$ which is directly attributable to the topological charge $\ell$ of the beam. The value of this charge denotes the number of $2 \pi$ phase changes around the singular point. The optical field typically has a radial dependence upon the value of the topological charge. ${ }^{2,3)}$ The term perfect vortex beam, first introduced by Ostrovsky et al., ${ }^{4)}$ refers to a vortex beam that retains a constant intensity profile and radius regardless of topological charge. Generally such vortex fields have potential applications for optical manipulation of mesoscopic particles and within the field of quantum gases ${ }^{5)}$ though to date, such studies have mostly been confined to those where the topological charge of the optical vortex in question has a dependency upon radius. ${ }^{3,6)}$ We recently generated a 'perfect' vortex field and investigated the dynamics of trapped particles. ${ }^{7)}$ In this paper, we extend that study to expound the detailed procedure of experimentally creating a 'perfect' vortex beam. In particular, we detail how we load and observe a single trapped particle to probe the local OAM density in such a vortex beam in order to improve the beam quality to attain a uniform OAM density in the optical field in situ. This present study thus aims to clarify the link between the macroscopic angular momentum transferred to the trapped particle and the state of the perfect vortex beam. In short, we find that uniform motion of the particle in the perfect vortex beam can only be achieved when the

*E-mail address: mc225@st-andrews.ac.uk 


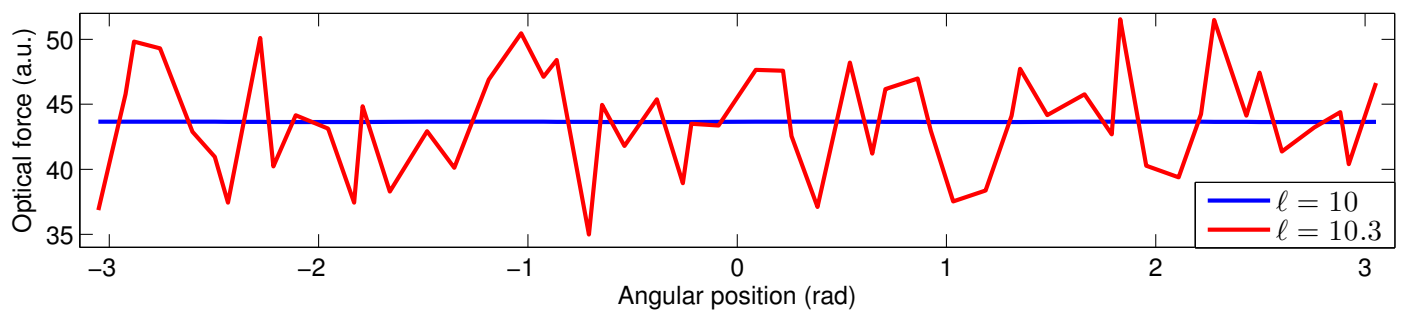

Fig. 1. (Color online) Numerical simulations of optical forces on a single trapped particle in a perfect vortex ring with $\ell=10$ and $\ell=10.3$.

beam is in a pure orbital angular momentum state which in the perfect case corresponds to integer topological charge.

\section{Model}

Firstly, to highlight the link between the trapped particle motion and the orbital angular momentum of the perfect vortex beam we consider the motion of a particle in the associated field. ${ }^{8)}$ To link directly to the experiment we consider $3 \mu \mathrm{m}$ polystyrene spheres dispersed in heavy water. The trapped transparent particle can be modelled as a non-absorbing, apertured ball lens. Based on the paraxial equation of diffraction, optical forces can be determined by a Maxwell stress tensor model. Adapting this model to the perfect vortex beam, we know that the particle motion speed is directly decided by the OAM density as $\ell \hbar$. The angular speed of a trapped particle in a vortex beam with different $\ell$ can be determined by its experienced optical force which is shown in Fig. 1. A vortex field with integer $\ell$ then delivers constant azimuthal velocity while non integer $\ell$ does not. Although we can 'smooth' and thus overcome the main hot spot in the field by uniformly distributing the non-integer $\ell$ around the vortex, the particle motion (and thus the optical force) still varies along the ring as shown by the red curve in Fig. 1. The experimental procedure outlined in this paper aims to achieve a constant azimuthal particle velocity and consequently this implies solely using light fields with integer $\ell$.

\section{Experimental setup}

The experimental optical system (Fig. 2) used for these studies has already been elaborated in our previous paper. ${ }^{7}$ ) To obtain a uniform ring profile, we first need to remove most of phase aberrations in the optical system by applying the SLM wavefront correction as introduced in reference. ${ }^{9)}$ As it can be compared in Fig. 3 (a) and (b), a high order Bessel beam with better beam quality can be obtained in the Fourier plane of the SLM.

However, there are still some hot spots (intensity peaks) on the beam profile in the trapping plane as shown in Fig. 3 (c) after wavefront correction. These unavoidable hot spots will stop the particle in the potential wells, which is a quite common problem in any single particle 


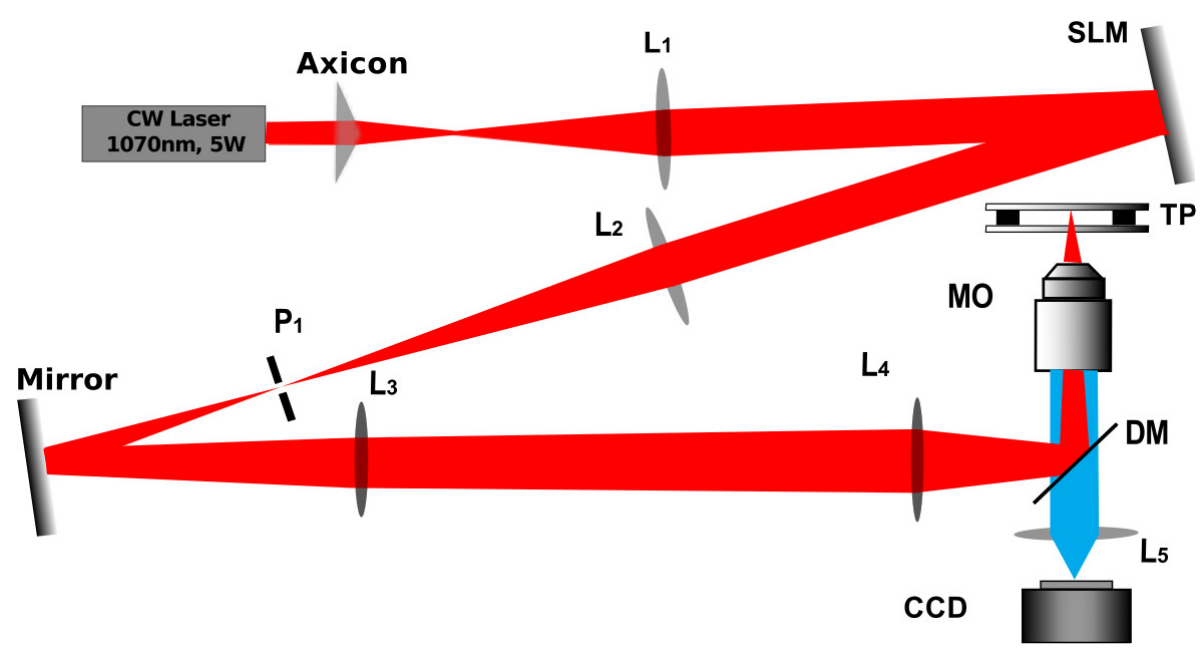

Fig. 2. (Color online) Schematic of the experimental setup used for trapping with a perfect vortex beam. Axicon apex angle is $\left.178^{\circ}\right) . L_{1} \sim L_{5}$ are lenses with $f_{1}=500 \mathrm{~mm}, f_{2}=135 \mathrm{~mm}, f_{3}=250 \mathrm{~mm}$, $f_{4}=50 \mathrm{~mm}$ and $f_{5}=200 \mathrm{~mm}$. $D M$ is a dichroic mirror reflecting the laser while transmitting the illumination light. $M O$ is a microscope objective (40X, NA=1.3). TP is the trapping plane. The standard part of LED white light illumination used to illuminate the trapping plane is not shown here however the optical path is illustrated by the blue beam.

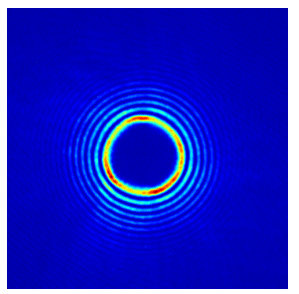

(a)

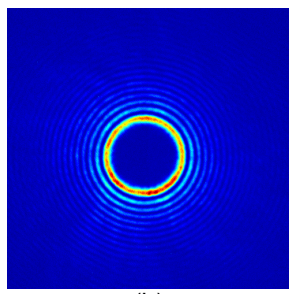

(b)

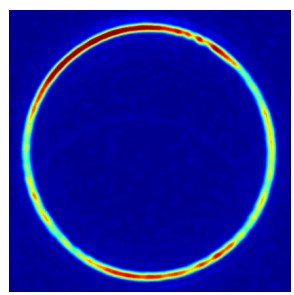

(c)

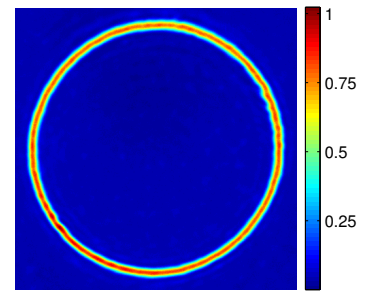

(d)

Fig. 3. (Color online) Beam profiles at the Fourier plane of SLM before (a) and after (b) wavefront correction. Beam profiles at trapping plane (TP) before (c) and after (d) wavefront correction. A high order vortex $(\ell=20)$ is at the beam centre.

trapping experiment. ${ }^{3,10)}$ Since the SLM is on the conjugation plane of trapping plane and it can do phase-only modulation, these intensity hot spots can be locally removed by reducing the local efficiency of phase modulation at the locations of hot spots. After such an intensity profile correction, the intensity uniformity of the beam is significantly improved as can be seen in Fig. 3 (d). A single trapped particle can have a continuous orbital rotation on such a vortex ring. 

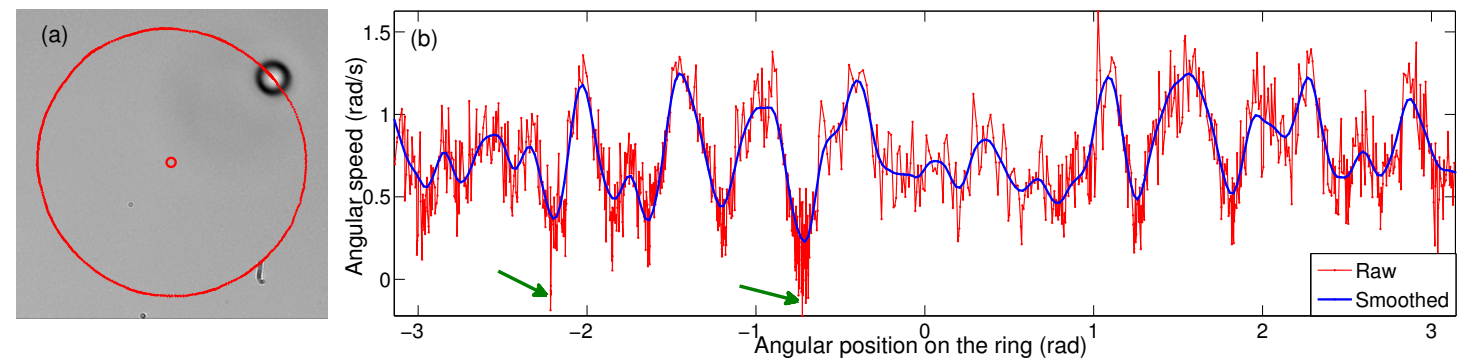

Fig. 4. (Color online) Rotation analysis of a single trapped particle. (a) shows one video frame of a trapped particle ( $3 \mu \mathrm{m}$ polystyrene spheres dispersed in heavy water). The small red circle shows the rotation centre and the red dots show the trace of the particle centre in video frames. (b) shows the measured particle angular velocity on the ring.

\section{Local OAM density correction based on a single trapped particle}

After all of the above corrections, it is straightforward to load a single particle onto the vortex ring if the particle concentration in the water solution is suitable. A trapped particle ( $3 \mu \mathrm{m}$ polystyrene spheres dispersed in heavy water) can now move along the vortex ring continuously. Its trajectory is as shown by the red trace in Fig. 4 (a). However, the angular velocity of such a trapped particle is not very uniform on the ring due to the local variations of OAM density. At some locations as pointed by the green arrows in Fig. 4 (b), the angular velocity even drops down to a negative value when the particle motion is mainly dominated by the Brownian motion.

Based on the knowledge that the particle rotation velocity is linearly dependent upon the OAM density, the angular velocity can be adjusted locally by increasing or decreasing the local OAM density. As shown in Fig. 5 (a), a local OAM density adjustment curve can be calculated based on the particle angular velocity. A phase correction mask then can be calculated in order to adjust the OAM density locally, as shown in Fig. 5 (b). Such a correction is performed in an iteration manner with a small amount of OAM adjustment in each iteration to avoid any sudden change.

\section{Results and discussions}

With the application of beam corrections discussed above, we experimentally create a perfect vortex beam which has the uniform intensity profile regardless of its topological charge. Phase, amplitude and local OAM density correction are applied in situ to improve the uniformity of OAM density in a perfect vortex beam. For a single trapped particle in a perfect vortex beam, we can achieve smooth rotation speed over the vortex ring, as shown in Fig. 6 . The importance of the work described in this paper lies in the novel use of the perfect vortex beam for applications of optical manipulation. This may be used for applications including 
Number of iterations: 5

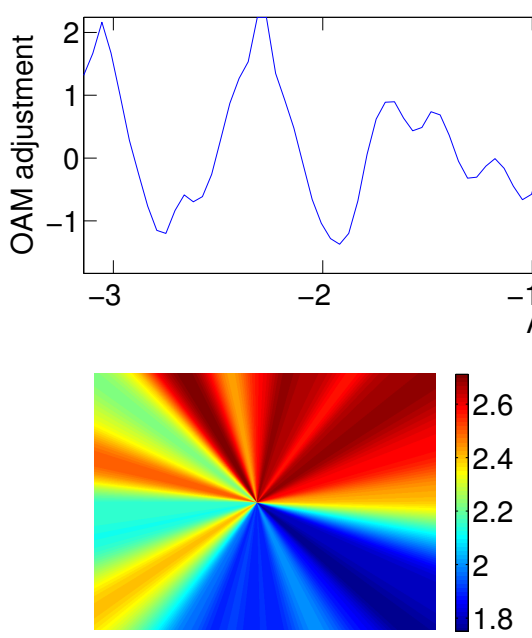

(b)

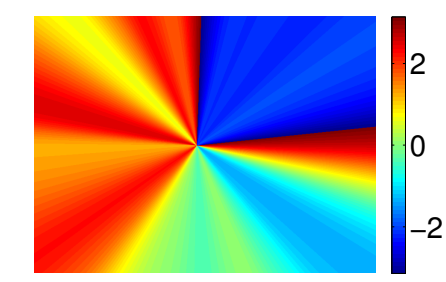

(c) (a)
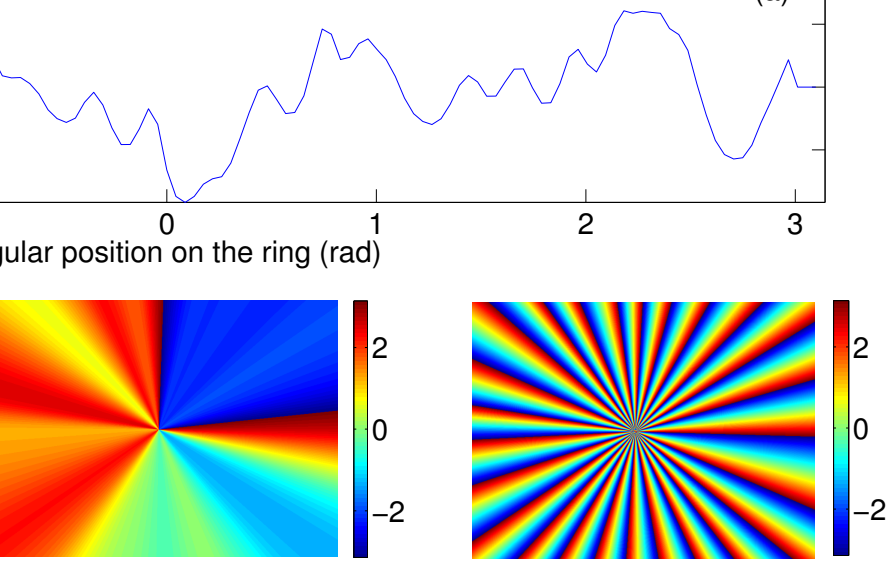

(d)

Fig. 5. (Color online) Fifth iteration of the iterative local OAM density correction. (a) shows the local OAM adjustment. (b) shows the correction phase calculated from (a). (c) shows the accumulated phase mask used for local OAM density correction from previous iterations. (d) shows a corrected vortex phase mask.

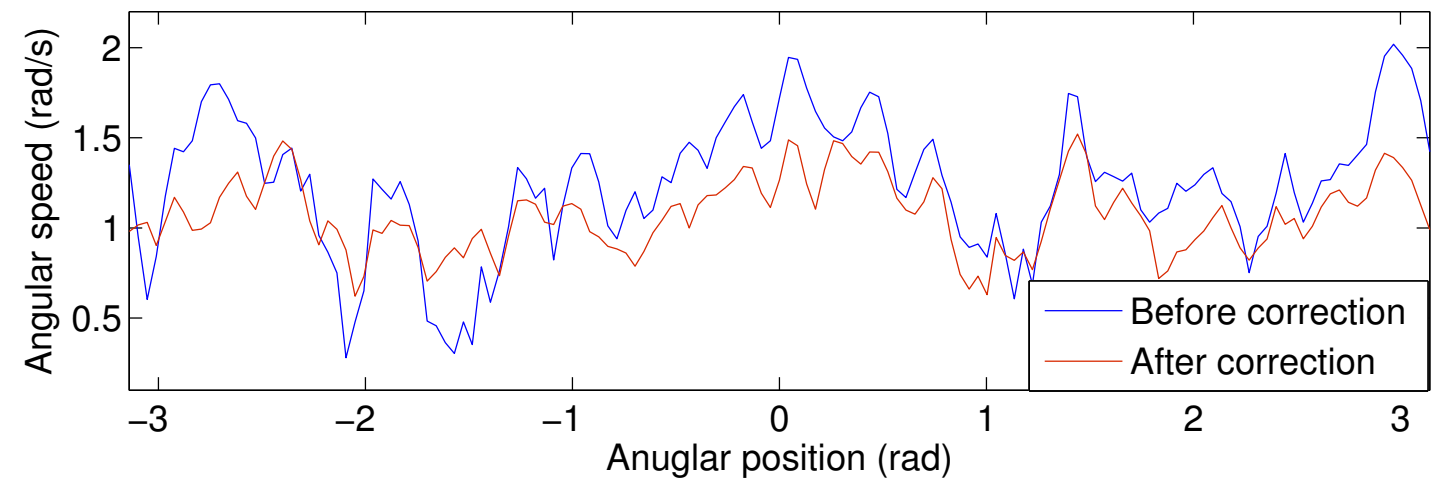

Fig. 6. (Color online) Angular velocity of a single trapped partile on a perfect vortex beam.

micromixing and moving microparticles on complex trajectories.

\section{Acknowledgment}

The authors would like to thank the UK Engineering and Physical Sciences Research Council (EPSRC) for funding. 


\section{References}

1) L. Allen, M. W. Beijersbergen, R. C. Spreeuw and J. P. Woerdman: Phys. Rev. A 45 (1992) 8185 .

2) N. B. Simpson, L. Allen and M. J. Padgett: J. Mod. Opt. 43 (1996) 2485.

3) J Curtis and D.G. Grier: Physical Review Letters 90 (2003) 133901.

4) A. S. Ostrovsky, C. Rickenstorff-Parrao and V. Arrizón: Opt. Lett. 38 (2013) 534.

5) M. F. Andersen, C. Ryu, P. Cladé, V. Natarajan, A. Vaziri, K. Helmerson and W. D. Phillips: Phys. Rev. Lett. 97 (2006) 170406.

6) V. Garces-Chavez, D. McGloin, M.J. Padgett, W. Dultz,,H. Schmitzer and K. Dholakia: Phys. Rev. Lett.91 (2003) 093602.

7) M. Chen, M. Mazilu, Y. Arita, E. M. Wright and K. Dholakia: Opt. Lett. 38 (2013) 4919.

8) J. Baumgartl, T. Čižmár, M. Mazilu, and V.C. Chan, A. E. Carruthers, B. A. Capron, W. McNeely, E. M. Wright, K. Dholakia: Opt. Express 18 (2010) 17130.

9) T. Čižmár, M. Mazilu, and K. Dholakia: Nat. Photonics 4 (2010) 388.

10) S. H. Tao, X.-C. Yuan and J. Lin: Opt. Express 13 (2005) 7726. 